\title{
ATMOSPHERIC RADIOCARBON CALIBRATION BEYOND 11,900 CAL BP FROM LAKE SUIGETSU LAMINATED SEDIMENTS
}

\author{
Hiroyuki Kitagawa \\ Institute for Hydrospheric-Atmospheric Sciences, Nagoya University, Furo-cho, Chikusa-ku, Nagoya, 464-8601, Japan. \\ Email: Kitagawa@ihas.nagoya-u.ac.jp. \\ Johannes van der Plicht \\ Centre for Isotope Research, Groningen University, Nijenborgh 4, 9747 AG Groningen, the Netherlands. \\ Email: plicht@phys.rug.nl.
}

\begin{abstract}
This paper presents an updated atmospheric radiocarbon calibration from annually laminated (varved) sediments from Lake Suigetsu (LS), central Japan. As presented earlier, the LS varved sediments can be used to extend the radiocarbon time scale beyond the tree ring calibration range that reaches $11,900 \mathrm{cal} \mathrm{BP}$. We have increased the density of ${ }^{14} \mathrm{C}$ measurements for terrestrial macrofossils from the same core analyzed previously. The combined data set now consists of 333 measurements, and is compared with other calibration data.
\end{abstract}

\section{INTRODUCTION}

The latest radiocarbon calibration curve (INTCAL98; Stuiver et al. 1998) was produced by combining several data sets of dendrochronologically dated tree rings for the Holocene, and uranium-thorium (U-Th) dated corals and marine sediments for the Glacial. Using the calibration data set and appropriate computer programs, the conversion of radiocarbon- to calibrated ages is now possible for $24,000-0$ cal BP (Before Present, 0 cal BP $=\mathrm{AD}$ 1950). To generate the atmospheric ${ }^{14} \mathrm{C}$ calibration curve before 11,900 cal BP, however, only a limited number of marine data with an assumed past marine reservoir age has been used.

Atmospheric ${ }^{14} \mathrm{C}$ calibration data with higher resolution for the period before $11,900 \mathrm{cal} \mathrm{BP}$ can be obtained from laminated sediments. The terrestrial macrofossils (e.g. leaves, branches, and insects) can be dated "absolutely" by counting varve numbers. They can overcome the uncertain assumptions for past marine reservoir ages, and can produce an atmospheric calibration curve with much higher resolution.

We have measured ${ }^{14} \mathrm{C}$ dates for terrestrial macrofossils from a long sequence of varved sediments from Lake Suigetsu (Kitagawa and van der Plicht 1998a, 1998b). Recently, we increased the density of ${ }^{14} \mathrm{C}$ measurements for terrestrial macrofossils from the same core analyzed previously. Combining the previous and new data sets, we have investigated the fine structure of the atmospheric ${ }^{14} \mathrm{C}$ calibration curve before $11,900 \mathrm{cal} \mathrm{BP}$. The ${ }^{14} \mathrm{C}$ calibration data from the LS varved sediments are presented here and compared with other calibration records.

\section{METHODS}

\section{Radiocarbon Dating}

${ }^{14} \mathrm{C}$ dating has been performed on terrestrial macrofossils (leaves, branches and insects) from the upper 35-m section of a single 75-m-long core (lab code SG) collected in 1993. All macrofossils used in this study were single pieces retaining its original form, in order to exclude the possibility of reworked material from the surroundings of the lake. To minimize potential contamination, we applied a strong acid-alkali-acid treatment to all the samples.

${ }^{14} \mathrm{C} /{ }^{12} \mathrm{C}$ and ${ }^{13} \mathrm{C} /{ }^{12} \mathrm{C}$ ratios were measured at the Groningen AMS facility (van der Plicht et al. 1995; Wijma and van der Plicht 1997) during 1994-1998. The background was determined by measuring 
fossil macrofossils collected from deep layers of the same core, with ages in the range 90-100 ka estimated by the tephra chronology of the LS core (Takemura et al. 1994). The average blank correction for the larger samples $(>0.7 \mathrm{mg}$ of carbon) is $0.30 \pm 0.03(1 \sigma)$ percent modern carbon. For smaller samples, we applied a mass-dependent correction based on the results from the ${ }^{14} \mathrm{C}$-free macrofossils. Duplicate measurements were averaged. The agreement between the previous and new data is excellent. The numbers are listed in Table 1 (see Appendix).

\section{Varve Chronology}

The 29,100-yr-long varve chronology in the section 10.42-30.45 $\mathrm{m}$ has been constructed using image analysis of around 1500 high resolution digital pictures (Kitagawa and van der Plicht 1998a, 1998b). This was done using the SG core and two short piston cores. The Sakate ash layer (varve nr 9895 , corresponding age $18,725 \mathrm{cal} \mathrm{BP}$ ) was recognized in the deepest part of the short piston cores and $18.67-\mathrm{m}$ deep in the SG cores. The tentative varve chronology produced from the SG core was reassessed based on the observation of the younger sediments above the Sakate ash layer. However, the LS varve chronology of the deeper section below the Sakate ash layer was produced by the varve counting of a single SG core. Beyond $18.8 \mathrm{ka}$ cal BP, the accuracy in the LS varve chronology would become worse, and these ages quoted in this paper should be considered as minimum ages.

\section{Absolute Age Determination and Its Uncertainty}

The absolute age of the LS floating varve chronology has been determined by wiggle-matching 22 ${ }^{14} \mathrm{C}$ dates from the younger part of the LS sediment to the revised German oak ${ }^{14} \mathrm{C}$ calibration curve (Spurk et al. 1998; Kromer and Spurk 1998). The previous matching (Kitagawa and van der Plicht 1988a, 1998b) is not revised, even with new data set.

The mean deviation of ${ }^{14} \mathrm{C}$ between our LS data and the revised oak data is $60 \pm 130{ }^{14} \mathrm{C}$ yr $(1 \sigma$ level). Omitting four outliers (using a $2 \sigma$ criterion) yields $55 \pm 100{ }^{14} \mathrm{C}$ yr. Likewise, we compared the LS ${ }^{14} \mathrm{C}$ calibration data with the combined German oak and German pine data (Spurk et al. 1998; Kromer and Spurk 1998). The mean deviation is then $40 \pm 170{ }^{14} \mathrm{C}$ yr $(\mathrm{n}=54)$, and $-5 \pm 100{ }^{14} \mathrm{C}$ yr when nine outliers are omitted. The apparent deviations might be caused by reworked macrofossils in the LS sediments and/or a blank correction problem. But except for a few outliers, the LS calibration data agree very well with the tree-ring curve.

The uncertainty in the absolute age estimation of the LS varve chronology mainly comes from two sources: 1) the varve chronology itself, and 2) the determination of the absolute age by wiggle matching the younger part of the sediment to the tree-ring curve. Since the detectability of the varve depends on the quality of the lamination, it is not straightforward to estimate the uncertainty in the LS varve chronology. Based on duplicate counting of selected sections (about $10 \%$ of the 29,100 -yrlong varve chronology), we estimate the uncertainty to be less than $1.5 \%$. In order to construct a more precise LS varve chronology, microscopic observation of thin sections will be performed in the near future. Another uncertainty in the LS varve chronology is caused by possibly incomplete sampling. The SG core was sampled for every $90-\mathrm{cm}$-long section from one drilling hole. The comparison with short piston cores suggests that the sampling does not cause critical loss of varves: typically $0-2 \mathrm{~cm}$ to a maximum of $3 \mathrm{~cm}$ for every sampling of about $90 \mathrm{~cm}$, corresponding to about 2030 and $50 \mathrm{yr}$ for the Holocene and the Late Glacial, respectively. However, the sampling loss causes an accumulation error in the LS varve chronology older than about 19,920 cal BP, corresponding to a depth of $19.39 \mathrm{~m}$ in the SG core. 


\section{RESULTS AND COMPARISON WITH OTHER RECORDS}

\section{Deglaciation Period}

The updated atmospheric ${ }^{14} \mathrm{C}$ calibration dataset from the LS varved sediments is compared with INTCAL98 (Figure 1). Back to $12.5 \mathrm{ka}$ cal BP, the LS data agree in general with INTCAL98, which is constructed from dendrochronologically dated tree rings, U-Th dated corals (Bard et al. 1998; Burr et al. 1998; Edwards et al. 1993) and marine sediments from the Cariaco basin (Hughen et al. 1998; Stuiver et al. 1998). Our LS calibration dataset also agrees well with new data from varved sediments of Lake Gościąż in Poland (Goslar et al. 2000a, 2000b), where a ${ }^{14} \mathrm{C}$ plateau is observed at $10,400 \mathrm{BP}$ (between 11.8 and $12.2 \mathrm{ka}$ cal $\mathrm{BP}$ ) and a rapid increase in ${ }^{14} \mathrm{C}$ age to $12.5 \mathrm{ka}$ cal $\mathrm{BP}$.

Before $12.5 \mathrm{ka}$ cal BP, there seems to be a systematic age offset by about $200{ }^{14} \mathrm{C}$ yr (Stuiver et al. 1998). It is possible that this is caused by an underestimation of about 200 varves at $12-13 \mathrm{ka}$ cal BP. However, a similar age offset has been observed in Lake Gościąż (Goslar et al. 2000a). We note that at present we have no indication or evidence for missing varves in this time interval.

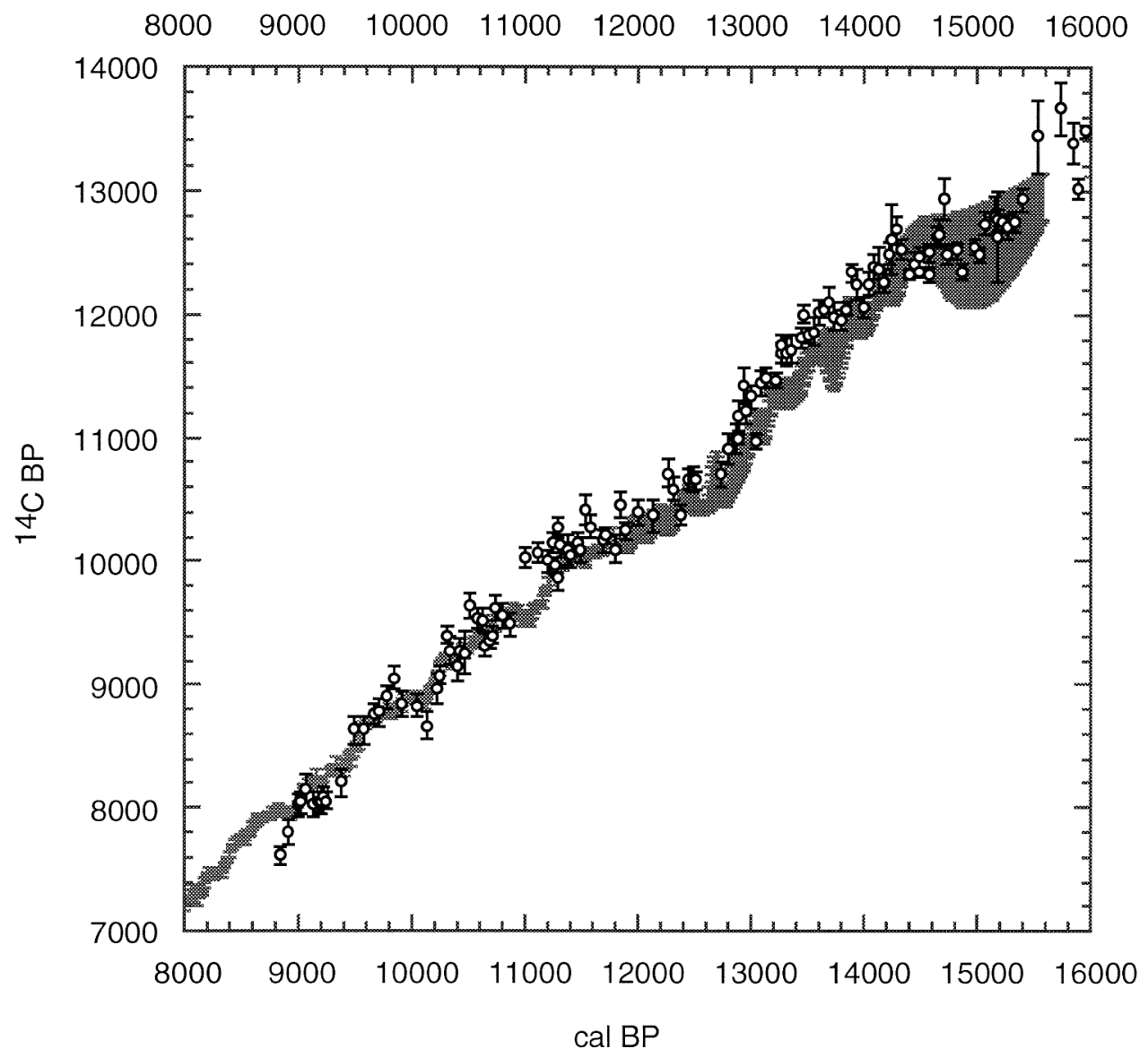

Figure 1 Comparison of atmospheric ${ }^{14} \mathrm{C}$ calibration from the varved sediments of Lake Suigetsu (LS) between 8000 and 16,000 cal BP (open circles with $\pm 1 \sigma$ error bar) with INTCAL9 8 calibration curve (shadowed by $\pm 1 \sigma$; Stuiver et al. 1998). 
Another possible explanation is the uncertainty of the marine reservoir correction $(\mathrm{R})$ applied in INTCAL98. In the marine-derived section of INTCAL98, the following assumptions were made: 1) $R$ remains constant for each individual site, and 2) for the period before $10 \mathrm{ka} \mathrm{cal} \mathrm{BP,} \mathrm{R}$ in the whole tropical surface ocean had a constant value of $500{ }^{14} \mathrm{C} \mathrm{yr}$ (and $400{ }^{14} \mathrm{C} \mathrm{yr}$ for samples younger than $10 \mathrm{ka}$ cal BP). If the R values used in INTCAL98 are corrected to the original site-specific reservoir corrections of the corals $\left(300{ }^{14} \mathrm{C}\right.$ yr for Tahiti and Mururoa and $400{ }^{14} \mathrm{C}$ yr for Barbados; Bard et al. 1998), the systematic age offset decreases to the error range of the LS varve chronology.

Although there are still uncertainties in the absolute age axis of our ${ }^{14} \mathrm{C}$ calibration as well as in the ${ }^{14} \mathrm{C}$ age axis of INTCAL98 before $12 \mathrm{ka}$ cal BP, our data show periods of a rather constant ${ }^{14} \mathrm{C}$ age (plateaux) at 11.6, 12.1, and 12.5 ka BP. This is consistent with the data from Lake Gościąz (Goslar et al. 2000a).

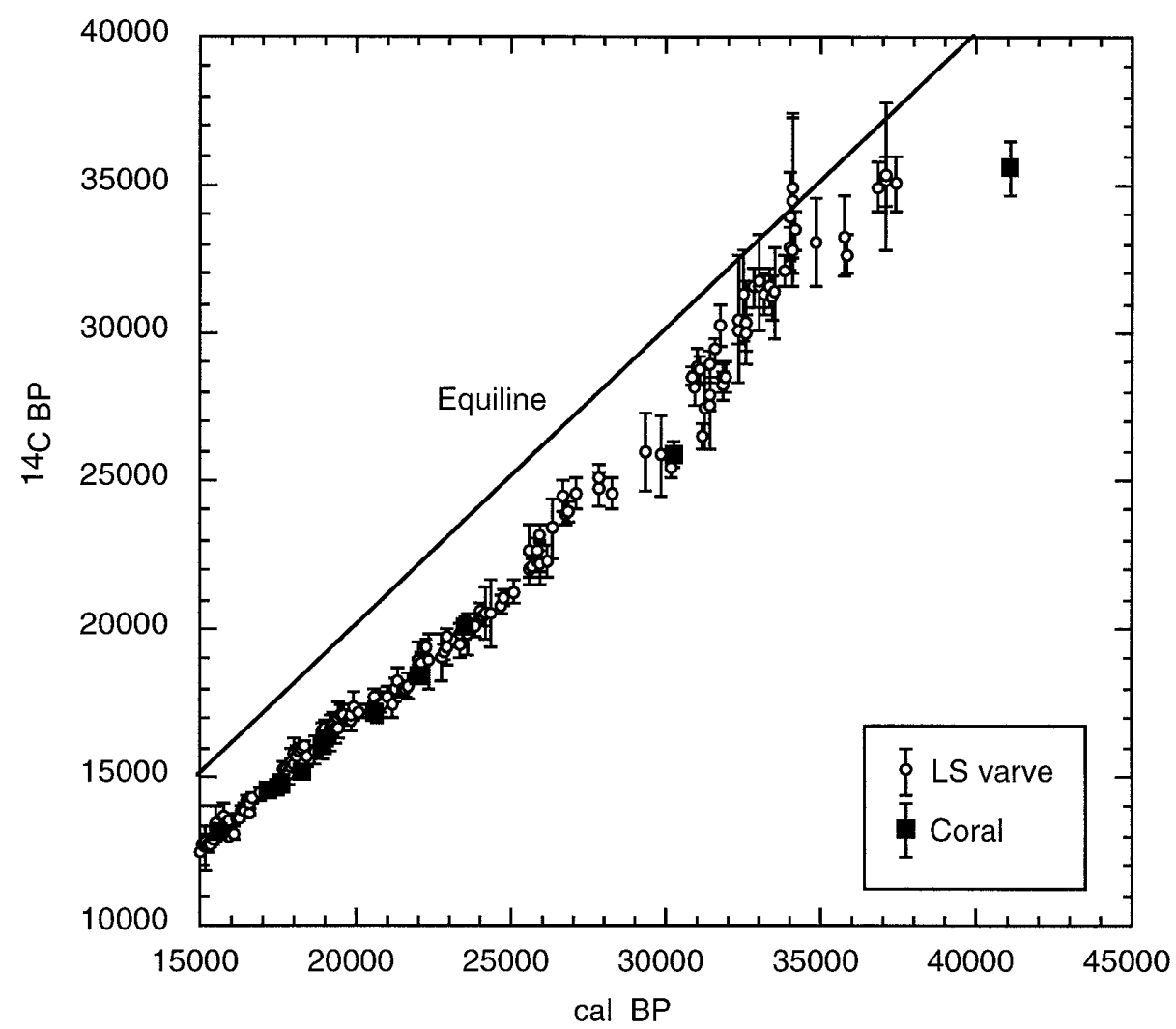

Figure 2 Radiocarbon calibration data from the varved sediments of Lake Suigetsu and U-Th dated corals (Bard et al. 1998) between 15,000 and 41,000 cal BP. Note that the error bars are $\pm 2 \sigma$.

\section{Full Glacial Period}

The older part of the $\mathrm{LS}{ }^{14} \mathrm{C}$ calibration dataset is compared with calibration data obtained from corals (Figure 2). Between 15,000 and around 24,000 cal BP, the long-term trend of the LS calibration agrees in general with the extended atmospheric calibration curve (INTCAL98) obtained from the U-Th dated corals (Bard et al. 1998), confirming the long-term increasing difference between ${ }^{14} \mathrm{C}$ and calendar ages. This trend agrees with the available calibration data obtained by cross-calibration 
of stable isotope ratios $\left({ }^{18} \mathrm{O}\right.$ from planktonic foraminifera) in North Atlantic cores with the Greenland GISP2 ice core (Voelker et al. 1998), U-Th age based calibration of South African stalagmites (Vogel and Kronfeld 1997) and Lake Lisan sediments in the northern Jordan Valley (Schramm et al. 2000; Stein et al. 2000).

Before $24 \mathrm{ka}$ cal BP, Bard et al.(1988) report two additional calibration datapoints at 30 and $41 \mathrm{ka}$ cal BP, suggesting that the age difference between ${ }^{14} \mathrm{C}$ and calibrated timescales increase to 3000 4000 and $4000-6000{ }^{14} \mathrm{C}$ yr, respectively. This large difference is confirmed by ${ }^{14} \mathrm{C}$ calibration data, obtained from U-Th dated sediments of Lake Lisan (Schramn et al. 2000). However, our data for Lake Suigetsu show a very different trend, suggesting a decrease of the ${ }^{14} \mathrm{C} /$ calendar age difference between 30 and $35 \mathrm{ka}$ cal BP. The precise calendar age determination for the LS varved sediment becomes more difficult with increased age because we reconstructed the LS varve chronology from one single core. Furthermore, possible contamination becomes more critical for older and smaller samples. The older part of the LS ${ }^{14} \mathrm{C}$ calibration curve remains still tentative, and additional work is needed to confirm the ${ }^{14} \mathrm{C}$ calibration in this age range.

\section{Fine Structure of the Glacial Calibration}

The time resolution of the LS calibration dataset for the Glacial period permits the investigation of fine structure in the atmospheric ${ }^{14} \mathrm{C}$ calibration curve. This curve can be strongly influenced by changes in ${ }^{14} \mathrm{C}$ production as well as by rearrangements in equilibrium between major $\mathrm{C}$ reservoirs (atmosphere, ocean and biosphere). For example, our data documents three periods of rather constant ${ }^{14} \mathrm{C}$ age at $12.5,17.2$, and possibly $25 \mathrm{ka} \mathrm{BP}$, recognized at $14.3-15.0,20.0-22$, and $28-30 \mathrm{ka}$ cal BP, respectively. Stuiver et al. (1998) suggest possible ${ }^{14} \mathrm{C}$ age plateaux during the Glacial, related to paleo-oceanic changes. Further discussions of the possible century- and millennium-scale fluctuations recognized in our Lake Suigetsu calibration data will be reported elsewhere.

\section{CONCLUSION}

The long sequence of varved sediments from Lake Suigetsu (Japan) permits an unique opportunity to establish a high-resolution atmospheric ${ }^{14} \mathrm{C}$ calibration curve back to 45,000 years or more. In general, varve-counting dating is only possible if the record is truly continuous; i.e. there is no hiatus or the hiatus is exactly known in time. Indeed the varve chronologies from Sweden (Wohlfarth 1996), Holzmaar in Germany (Hajdas et al. 1995), and Soppensee in Switzerland (Hajdas et al. 1993) have been shifted by several hundred years toward an older age. For Lake Suigetsu, independent checks of varve- ${ }^{14} \mathrm{C}$ calibration still need to confirm our ${ }^{14} \mathrm{C}$ calibration curve, in particular beyond $24,000 \mathrm{cal}$ BP. Nevertheless, some fine structure during the glacial has been partly revealed.

\section{ACKNOWLEDGMENTS}

All the ${ }^{14}$ C AMS measurements were performed during 1995-1998 at Groningen AMS Facility (the Netherlands). We thank Stef Wijma for precise AMS ${ }^{14} \mathrm{C}$ measurements.

\section{REFERENCES}

Bard E, Arnold M, Hamelin B, Tisneart-Laborde, Cabioch G. 1998. Radiocarbon calibration by means of mass spectrometric ${ }^{230} \mathrm{Th} /{ }^{234} \mathrm{U}$ and ${ }^{14} \mathrm{C}$ ages of coral: An updated database including samples from Barbados, Mururoa and Tahiti. Radiocarbon 40(3):108592.

Burr GS, Beck JW, Taylor FW, Récy J, Edwards RL, Ca- bioch G, Corrège T, Donahue DJ, O’Malley JM. 1998. A high-resolution radiocarbon calibration between 11,700 and $12,400 \mathrm{cal} \mathrm{BP}$ derived from ${ }^{230} \mathrm{Th}$ ages of corals from Espiritu Santo Island, Vanuatu. Radiocarbon 40(3):1085-92.

Edwards RL, Beck JW, Burr GS, Donahue DL, Chappell JMA, Bloom AL, Druffel ERM, Taylor FW. 1993. A 
large drop in atmospheric ${ }^{4} \mathrm{C} /{ }^{12} \mathrm{C}$ and reduced melting in the Younger Dryas, documented with ${ }^{230} \mathrm{Th}$ ages of corals. Science 260:962-7.

Goslar T, Arnold M, Tisnerat-Laborde N, Czernik J, Wieckowski K. 2000a. Variation of Younger Dryas atmospheric radiocarbon explicable without ocean circulation changes. Nature 403:877-80.

Goslar T, Arnold M, Tisnerat-Laborde, Hatte C, Paterne M, Ralska-Jasiewiczowa M. 2000b. Radiocarbon calibration by means of varves versus ${ }^{14} \mathrm{C}$ ages of terrestrial macrofossils from Lake Gościąż and Lake Perespilno, Poland. Radiocarbon. This issue.

Hajdas I, Ivy SD, Beer J, Bonani G, Imboden D, Lotter AF, Sturm M, Suter M. 1993. AMS radiocarbon dating and varve chronology of Lake Soppensee: 6000 to $12000{ }^{14} \mathrm{C}$ years BP. Climatic Dynamics 9:107-16.

Hajdas I, Zolischka B, Ivy-Ochs SD, Beer J, Bonani, Leroy SAG, Negendank JW, Ramrath M, Suter M. 1995. AMS radiocarbon dating of annually laminated sediments from Lake Holzmaar, Germany. Quaternary Science Reviews 14:137-43.

Hughen KA, Overpeck JT, Lehman SJ, Kashgrian M, Southon J, Peterson LC, Alley R, Sigman DM. 1998. Deglacial changes in ocean circulation from an extended radiocarbon calibration. Nature 391:65-8.

Kitagawa H, van der Plicht J. 1998a. Atmospheric radiocarbon calibration to $45,000 \mathrm{yr}$ BP: Late Glacial fluctuations and cosmogenic isotope production. Science 279:1178-90.

Kitagawa H, van der Plicht J. 1998b. A 40,000-year varve chronology from Lake Suigetsu, Japan: extension of the radiocarbon calibration curve. Radiocarbon 40(1):505-15.

Kromer B, Spurk M. 1998. Revision and tentative extension of the tree-ring based ${ }^{14} \mathrm{C}$ calibration, 9200 11,855 cal BP. Radiocarbon 40(3):1117-25.

Schramm A, Stein M, Goldstein SL. 2000. Calibration of the ${ }^{14} \mathrm{C}$ time scale to $>40$ ka by ${ }^{234} \mathrm{U}-{ }^{230} \mathrm{Th}$ dating of Lake Lisan sediments (last glacial Dead Sea). Earth and Planetary Science Letters 149:121-9.

Stein M, et al. 2000. Radiocarbon calibration beyond the dendrochronology range. Radiocarbon. This issue.

Stuiver M, Reimer PJ, Bard E, Beck JW, Burr GS, Hughen KA, Kromer B, McCormac G, van der Plicht J, Spurk M. 1998. INTCAL98 radiocarbon age calibration, 24,000-0 cal BP. Radiocarbon 40(3):104183.

Spurk M, Friedrich M, Hofmann J, Remmele S, Frenzel B, Leuschner HH, Kromer B. 1998. Revisions and extensions of the Hohenheim oak and pine chronologies: new evidence about the timing of the Younger Dryas/ Preboreal transition. Radiocarbon 40(3):1107-16.

Takemura K, Kitagawa H, Hayashida A, Yasuda Y. 1994. Sediment facies and chronology of core samples from lake Mikata, Lake Suigetsu and Kurota Lowland, central Japan: sedimentary environment in Mikata Lowland since the last interglacial time. Journal of Geography 103(3):233-42.

van der Plicht J, Aerts A, Wijma S, Zondervan A. 1995. First results from the Groningen AMS facility. Radiocarbon 37(2):657-61.

Vogel JC, Kronfeld J. 1997. Calibration of radiocarbon dates for the late Pleistocene using U/Th dates on stalagmites. Radiocarbon 39(1):27-32.

Voelker AHL, Sarnthein M, Grootes PM, Erlenkeuser H, Laj C, Mazaud A, Nadeau M-J, Schleicher M. 1998. Correlation of marine ${ }^{14} \mathrm{C}$ ages from the Nordic sea with GISP2 isotope record implications for radiocarbon calibration beyond $25 \mathrm{ka}$ BP. Radiocarbon 40(1): 517-34.

Wijma S, van der Plicht S. 1997. The Groningen AMS tandetron. Nuclear Instruments and Method in Physics Research B123: 218-20.

Wohlfarth B. 1996. The chronology of the last termination: a review of radiocarbon dated, high-resolution terrestrial stratigraphy. Quaternary Science Reviews $15: 267-84$ 


\section{APPENDIX}

Table 1 Varve and ${ }^{14} \mathrm{C}$ chronologies of varved sediments from Lake Suigetsu. In the first column, I shows already reported data (Kitagawa and van der Plicht 1998a, 1998b); II and III show the new data measured in March, 1998 and August, 1998, respectively. Duplicate measurements are averaged.

\begin{tabular}{|c|c|c|c|c|c|c|c|}
\hline \multirow[b]{2}{*}{ ID } & \multirow[b]{2}{*}{ Sample } & \multicolumn{2}{|c|}{ Depth $(\mathrm{cm})$} & \multicolumn{2}{|c|}{ Varve age (cal BP) } & \multirow{2}{*}{$\frac{{ }^{14} \mathrm{C} \text { age }}{(\mathrm{BP} \pm 1 \sigma)}$} & \multirow{2}{*}{$\begin{array}{l}\text { Lab code } \\
\text { GrA- }\end{array}$} \\
\hline & & Upper & Lower & Upper & Lower & & \\
\hline I & SG13D01 & 1042.0 & 1045.3 & 8828 & 8862 & $7610 \pm 70$ & 6234 \\
\hline I & SG13D04 & 1051.8 & 1055.0 & 8907 & 8931 & $7810 \pm 100$ & 2849 \\
\hline I & SG13D07 & 1061.5 & 1064.8 & 8984 & 9013 & $8020 \pm 90$ & 6233 \\
\hline I & SG13D08 & 1064.8 & 1068.0 & 9013 & 9050 & $8040 \pm 90$ & 6232 \\
\hline I & SG13D09 & 1069.1 & 1073.4 & 9050 & 9078 & $8150 \pm 110$ & 2839 \\
\hline I & SG13C03 & 1077.8 & 1081.0 & 9118 & 9138 & $8020 \pm 100$ & 2914 \\
\hline I & SG13C05 & 1084.3 & 1087.5 & 9158 & 9183 & $8050 \pm 80$ & 6235 \\
\hline I & SG13C06 & 1087.5 & 1090.8 & 9183 & 9207 & $8040 \pm 100$ & 6236 \\
\hline I & SG13C07 & 1090.8 & 1094.0 & 9207 & 9224 & $8090 \pm 90$ & 2840 \\
\hline I & SG13C09 & 1095.6 & 1098.3 & 9243 & 9263 & $8050 \pm 70$ & $2947 ; 2948$ \\
\hline I & SG13B05 & 1113.5 & 1118.4 & 9373 & 9402 & $8200 \pm 110$ & 2901 \\
\hline I & SG13A04 & 1126.5 & 1129.8 & 9481 & 9501 & $8640 \pm 110$ & 2842 \\
\hline I & SG14D03 & 1139.5 & 1142.7 & 9575 & 9600 & $8640 \pm 110$ & 2843 \\
\hline I & SG14D06 & 1149.2 & 1152.5 & 9646 & 9671 & $8770 \pm 80$ & 3087 \\
\hline I & SG14C01 & 1156.8 & 1160.1 & 9705 & 9727 & $8780 \pm 110$ & 2835 \\
\hline I & SG14C04 & 1166.6 & 1169.8 & 9769 & 9800 & $8900 \pm 90$ & 3085 \\
\hline I & SG14C06 & 1173.0 & 1176.3 & 9825 & 9848 & $9060 \pm 90$ & 3080 \\
\hline I & SG14B02 & 1182.2 & 1185.5 & 9892 & 9918 & $8850 \pm 110$ & 2844 \\
\hline I & SG14B07 & 1198.5 & 1201.7 & 10,030 & 10,055 & $8830 \pm 100$ & 3082 \\
\hline I & SG14A04 & 1211.5 & 1214.7 & 10,127 & 10,146 & $8670 \pm 110$ & 2890 \\
\hline I & SG15D01 & 1225.0 & 1228.4 & 10,213 & 10,239 & $8970 \pm 120$ & 3079 \\
\hline II & SG15D02 & 1228.4 & 1231.8 & 10,239 & 10,261 & $9070 \pm 70$ & 8184 \\
\hline III & SGD-012 & 1237.4 & 1238.6 & 10,307 & 10,316 & $9400 \pm 70$ & 10,243 \\
\hline I & SG15D05 & 1238.5 & 1241.9 & 10,316 & 10,350 & $9280 \pm 120$ & 2971 \\
\hline I & SG15D07 & 1245.3 & 1248.7 & 10,377 & 10,403 & $9150 \pm 120$ & 2845 \\
\hline I & SG15C01 & 1248.7 & 1252.1 & 10,403 & 10,425 & $9270 \pm 120$ & 2921 \\
\hline I & SG15C03 & 1255.5 & 1258.9 & 10,450 & 10,470 & $9260 \pm 180$ & 4585 \\
\hline I & SG15C06 & 1265.6 & 1269.0 & 10,510 & 10,532 & $9640 \pm 100$ & 2915 \\
\hline I & SG15C08 & 1272.4 & 1275.8 & 10,556 & 10,580 & $9540 \pm 80$ & 3081 \\
\hline I & SG15B02 & 1279.2 & 1282.6 & 10,603 & 10,626 & $9530 \pm 90$ & 2847 \\
\hline I & SG15B03 & 1282.6 & 1286.0 & 10,626 & 10,655 & $9320 \pm 90$ & 2944 \\
\hline II & SG15B05 & 1289.3 & 1292.7 & 10,680 & 10,706 & $9360 \pm 60$ & 8183 \\
\hline I & SG15B06 & 1292.7 & 1296.1 & 10,706 & 10,732 & $9410 \pm 80$ & 2913 \\
\hline I & SG15B07 & 1296.1 & 1299.5 & 10,732 & 10,758 & $9630 \pm 100$ & 2912 \\
\hline I & SG15A01 & 1303.5 & 1306.8 & 10,785 & 10,809 & $9560 \pm 110$ & 3083 \\
\hline I & SG15A04 & 1313.6 & 1317.0 & 10,857 & 10,880 & $9500 \pm 90$ & 2907 \\
\hline III & SGD-089 & 1326.2 & 1327.4 & 10,995 & 11,009 & $10,030 \pm 80$ & 10,260 \\
\hline I & SG16D06 & 1334.8 & 1338.6 & 11,102 & 11,144 & $10,080 \pm 90$ & 3086 \\
\hline I & SG16C01 & 1342.5 & 1345.9 & 11,180 & 11,215 & $10,010 \pm 100$ & 2904 \\
\hline III & SGD-109 & 1348.9 & 1350.0 & 11,243 & 11,253 & $10,150 \pm 80$ & 10,240 \\
\hline II & SG16C03 & 1349.2 & 1352.5 & 11,246 & 11,278 & $9960 \pm 80$ & 8182 \\
\hline I & SG16C04 & 1352.5 & 1355.8 & 11,278 & 11,303 & $9860 \pm 100$ & 2905 \\
\hline I & SG16C04 & 1352.5 & 1355.8 & 11,278 & 11,303 & $9860 \pm 100$ & 2905 \\
\hline III & SGD-114 & 1354.5 & 1355.7 & 11,293 & 11,301 & $10,280 \pm 90$ & 10,233 \\
\hline
\end{tabular}


Table 1 Varve and ${ }^{14} \mathrm{C}$ chronologies of varved sediments from Lake Suigetsu. In the first column, I shows already reported data (Kitagawa and van der Plicht 1998a, 1998b); II and III show the new data measured in March, 1998 and August, 1998, respectively. Duplicate measurements are averaged. (Continued)

\begin{tabular}{|c|c|c|c|c|c|c|c|}
\hline \multirow[b]{2}{*}{ ID } & \multirow[b]{2}{*}{ Sample } & \multicolumn{2}{|c|}{ Depth $(\mathrm{cm})$} & \multicolumn{2}{|c|}{ Varve age (cal BP) } & \multirow{2}{*}{$\frac{{ }^{14} \mathrm{C} \text { age }}{(\mathrm{BP} \pm 1 \sigma)}$} & \multirow{2}{*}{$\begin{array}{l}\text { Lab code } \\
\text { GrA- }\end{array}$} \\
\hline & & Upper & Lower & Upper & Lower & & \\
\hline I & SG16C05 & 1355.8 & 1359.7 & 11,303 & 11,336 & $10,130 \pm 100$ & 2911 \\
\hline I & SG16B01 & 1362.5 & 1365.8 & 11,358 & 11,384 & $10,100 \pm 130$ & 2961 \\
\hline I & SG16B02 & 1365.8 & 1369.2 & 11,384 & 11,414 & $10,060 \pm 100$ & 2838 \\
\hline I & SG16B04 & 1372.5 & 1375.8 & 11,447 & 11,474 & $10,150 \pm 100$ & 2917 \\
\hline I & SG16B05 & 1375.8 & 1379.1 & 11,474 & 11,506 & $10,100 \pm 100$ & 2916 \\
\hline III & SGD-138 & 1382.8 & 1383.3 & 11,539 & 11,545 & $10,410 \pm 120$ & 10,234 \\
\hline I & SG16A02 & 1385.3 & 1388.6 & 11,562 & 11,596 & $10,290 \pm 90$ & 3078 \\
\hline I & SG16A05 & 1395.2 & 1398.6 & 11,664 & 11,699 & $10,170 \pm 100$ & 2902 \\
\hline I & SG16A06 & 1398.6 & 1401.9 & 11,699 & 11,733 & $10,120 \pm 100$ & 2909 \\
\hline II & SG16A06 & 1398.6 & 1401.9 & 11,699 & 11,733 & $10,270 \pm 70$ & 8181 \\
\hline I & SG17D01 & 1408.0 & 1411.1 & 11,789 & 11,827 & $10,100 \pm 110$ & 2969 \\
\hline I & SG17D02 & 1411.1 & 1414.1 & 11,827 & 11,870 & $10,460 \pm 100$ & 2836 \\
\hline II & SG17D03 & 1414.1 & 1417.2 & 11,870 & 11,905 & $10,250 \pm 80$ & 1736 \\
\hline I & SG17D06 & 1423.3 & 1426.3 & 11,986 & 1,2023 & $10,400 \pm 110$ & 2970 \\
\hline I & SG17D10 & 1435.5 & 1438.0 & 12,129 & 12,157 & $10,370 \pm 130$ & 2981 \\
\hline I & SG17C03 & 1444.1 & 1447.2 & 12,240 & 12,282 & $10,710 \pm 110$ & 2837 \\
\hline I & SG17C04 & 1447.2 & 1450.2 & 12,282 & 12,322 & $10,590 \pm 100$ & 2913 \\
\hline I & SG17B01 & 1453.3 & 1456.3 & 12,352 & 12,383 & $10,380 \pm 90$ & 2906 \\
\hline II & SG17B03 & 1459.4 & 1462.4 & 12,421 & 12,461 & $10,670 \pm 80$ & 8179 \\
\hline I & SG17B04 & 1462.4 & 1465.5 & 12,461 & 12,500 & $10,670 \pm 100$ & 2848 \\
\hline II & SG17B05 & 1465.5 & 1468.5 & 12,500 & 12,537 & $10,660 \pm 70$ & 8178 \\
\hline I & SG17A02 & 1482.2 & 1485.3 & 12,718 & 12,754 & $10,700 \pm 100$ & 2908 \\
\hline I & SG17A04 & 1488.3 & 1491.4 & 12,782 & 12,805 & $10,920 \pm 130$ & 2920 \\
\hline I & SG17A07 & 1496.5 & 1498.0 & 12,851 & 12,864 & $11,000 \pm 130$ & 3077 \\
\hline $\mathrm{I}, \mathrm{II}$ & SG18E01 & 1498.0 & 1501.0 & 12,864 & 12,910 & $10,990 \pm 40$ & $4532 ; 8177$ \\
\hline III & SGD-248 & 1499.6 & 1500.6 & 12,887 & 12,905 & $11,180 \pm 130$ & 10,268 \\
\hline I & SG18E02 & 1501.0 & 1504.0 & 12,910 & 12,947 & $11,420 \pm 150$ & 5634 \\
\hline I & SG18E03 & 1504.0 & 1507.0 & 12,947 & 12,987 & $11,210 \pm 90$ & 5635 \\
\hline I & SG18E04 & 1507.0 & 1509.9 & 12,987 & 13,028 & $11,340 \pm 90$ & 5637 \\
\hline I & SG18E05 & 1509.9 & 1512.9 & 13,028 & 13,067 & $10,980 \pm 60$ & 4533 \\
\hline I & SG18E06 & 1512.9 & 1515.9 & 13,067 & 13,112 & $11,440 \pm 110$ & 5638 \\
\hline I & SG18E07 & 1515.9 & 1518.9 & 13,112 & 13,151 & $11,480 \pm 90$ & 5639 \\
\hline I & SG18D01 & 1521.4 & 1524.4 & 13,188 & 13,236 & $11,460 \pm 60$ & 4534 \\
\hline I & SG18D02 & 1524.4 & 1527.3 & 13,236 & 13,284 & $11,690 \pm 90$ & 5640 \\
\hline III & SGD-274 & 1525.8 & 1526.9 & 13,255 & 13,276 & $11,760 \pm 80$ & 10,232 \\
\hline II & SG18D03 & 1527.3 & 1530.3 & 13,284 & 13,338 & $11,700 \pm 120$ & 8190 \\
\hline II & SG18D04 & 1530.3 & 1533.3 & 13,338 & 13,394 & $11,720 \pm 110$ & 8139 \\
\hline III & SGD-284 & 1536.0 & 1537.0 & 13,435 & 13,454 & $11,810 \pm 80$ & 10,238 \\
\hline II & SG18D06 & 1536.3 & 1539.3 & 13,441 & 13,492 & $12,000 \pm 80$ & 1719 \\
\hline I & SG18C01 & 1539.3 & 1542.3 & 13,492 & 13,537 & $11,830 \pm 70$ & 5641 \\
\hline II & SG18C02 & 1542.3 & 1544.2 & 13,537 & 13,573 & $11,860 \pm 110$ & 8176 \\
\hline II & SG18C03 & 1544.2 & 1547.2 & 13,573 & 13,621 & $12,010 \pm 100$ & 8151 \\
\hline I,II & SG18C04 & 1547.2 & 1550.2 & 13,621 & 13,672 & $12,030 \pm 60$ & 4535 \\
\hline II & SG18C05 & 1550.2 & 1553.2 & 13,672 & 13,717 & $12,100 \pm 130$ & 8194 \\
\hline I & SG18B01 & 1553.2 & 1556.7 & 13,717 & 13,767 & $11,980 \pm 110$ & 5653 \\
\hline II & SG18B02 & 1556.7 & 1559.7 & 13,767 & 13,814 & $11,960 \pm 80$ & 8175 \\
\hline
\end{tabular}


Table 1 Varve and ${ }^{14} \mathrm{C}$ chronologies of varved sediments from Lake Suigetsu. In the first column, I shows already reported data (Kitagawa and van der Plicht 1998a, 1998b); II and III show the new data measured in March, 1998 and August, 1998, respectively. Duplicate measurements are averaged. (Continued)

\begin{tabular}{|c|c|c|c|c|c|c|c|}
\hline \multirow[b]{2}{*}{ ID } & \multirow[b]{2}{*}{ Sample } & \multicolumn{2}{|c|}{ Depth (cm) } & \multicolumn{2}{|c|}{ Varve age (cal BP) } & \multirow{2}{*}{$\frac{{ }^{14} \mathrm{C} \text { age }}{(\mathrm{BP} \pm 1 \sigma)}$} & \multirow{2}{*}{$\begin{array}{l}\text { Lab code } \\
\text { GrA- }\end{array}$} \\
\hline & & Upper & Lower & Upper & Lower & & \\
\hline I & SG18B03 & 1559.7 & 1562.6 & 13,814 & 13,865 & $12,040 \pm 60$ & 4536 \\
\hline II & SG18B04 & 1562.6 & 1565.6 & 13,865 & 13,914 & $12,330 \pm 70$ & 8147 \\
\hline I & SG18B05 & 1565.6 & 1568.6 & 13,914 & 13,967 & $12,250 \pm 130$ & 6206 \\
\hline I & SG18B06 & 1568.6 & 1572.1 & 13,967 & 14,023 & $12,050 \pm 90$ & 4537 \\
\hline I & SG18A01 & 1572.1 & 1575.1 & 14,023 & 14,067 & $12,250 \pm 100$ & 5642 \\
\hline II & SG18A02 & 1575.1 & 1578.1 & 14,067 & 14,116 & $12,380 \pm 100$ & 8189 \\
\hline II & SG18A03 & 1578.1 & 1581.0 & 14,116 & 14,159 & $12,360 \pm 190$ & 8191 \\
\hline I & SG18A04 & 1581.0 & 1584.0 & 14,159 & 14,198 & $12,270 \pm 100$ & 6202 \\
\hline II & SG18A05 & 1584.0 & 1587.0 & 14,198 & 14,238 & $12,490 \pm 90$ & 8148 \\
\hline I & SG18A06 & 1587.0 & 1589.0 & 14,238 & 14,267 & $12,610 \pm 300$ & 5654 \\
\hline II & SG19D01 & 1589.0 & 1592.1 & 14,267 & 14,316 & $12,680 \pm 120$ & 8150 \\
\hline II & SG19D02 & 1592.1 & 1595.3 & 14,316 & 14,366 & $12,520 \pm 80$ & 8143 \\
\hline I,II & SG19D03 & 1595.3 & 1598.4 & 14,366 & 14,421 & $12,320 \pm 50$ & $4539 ; 8185$ \\
\hline I & SG19D04 & 1598.4 & 1601.6 & 14,421 & 14,467 & $12,410 \pm 100$ & 6204 \\
\hline III & SGD-350 & 1602.4 & 1603.4 & 14,480 & 14,497 & $12,460 \pm 90$ & 10,231 \\
\hline I,II & SG19D05 & 1601.6 & 1604.7 & 14,467 & 14,514 & $12,350 \pm 50$ & $5643 ; 8188$ \\
\hline I & SG19D08 & 1601.6 & 1604.7 & 14,467 & 14,514 & $12,500 \pm 70$ & $5644 ; 8160$ \\
\hline $\mathrm{I}, \mathrm{II}$ & SG19D07 & 1607.8 & 1611.0 & 14,558 & 14,607 & $12,320 \pm 60$ & 4540 \\
\hline III & SGD-360 & 1612.7 & 1613.8 & 14,633 & 14,648 & $12,630 \pm 90$ & 10,239 \\
\hline II & SG19C01 & 1614.1 & 1617.2 & 14,651 & 14,702 & $12,660 \pm 110$ & 8156 \\
\hline III & SGD-364 & 1616.8 & 1617.9 & 14,695 & 14,713 & $12,940 \pm 160$ & 10,235 \\
\hline II & SG19C02 & 1617.2 & 1620.4 & 14,702 & 14,748 & $12,480 \pm 90$ & 8159 \\
\hline I & SG19C04 & 1623.5 & 1626.7 & 14,787 & 14,838 & $12,520 \pm 70$ & 5645 \\
\hline I & SG19C05 & 1626.7 & 1629.8 & 14,838 & 14,879 & $12,350 \pm 60$ & 4541 \\
\hline II & SG19C08 & 1636.1 & 1638.2 & 14,961 & 14,992 & $12,550 \pm 60$ & 8173 \\
\hline I & SG19B01 & 1638.2 & 1641.3 & 14,992 & 15,044 & $12,490 \pm 60$ & 4542 \\
\hline II & SG19B02 & 1641.3 & 1644.4 & 15,044 & 15,093 & $12,740 \pm 100$ & 8135 \\
\hline III & SGD-394 & 1647.8 & 1648.8 & 15,151 & 15,169 & $12,800 \pm 150$ & 10,242 \\
\hline I & SG19B04 & 1647.6 & 1650.7 & 15,148 & 15,202 & $12,630 \pm 370$ & 5646 \\
\hline III & SGD-395 & 1648.8 & 1649.8 & 15,169 & 15,187 & $12,770 \pm 90$ & 10,237 \\
\hline I & SG19B05 & 1650.7 & 1653.9 & 15,202 & 15,251 & $12,750 \pm 80$ & 4543 \\
\hline I & SG19B06 & 1653.9 & 1657.0 & 15,251 & 15,306 & $12,710 \pm 110$ & 6205 \\
\hline II & SG19B07 & 1657.0 & 1660.1 & 1,5306 & 15,370 & $12,750 \pm 80$ & 8140 \\
\hline II & SG19A01 & 1660.1 & 1663.3 & 15,370 & 15,427 & $12,930 \pm 90$ & 8136 \\
\hline I & SG19A03 & 1667.4 & 1670.6 & 15,501 & 15,555 & $13,440 \pm 300$ & 5648 \\
\hline I & SG20D01 & 1680.0 & 1683.3 & 15,713 & 15,764 & $13,670 \pm 220$ & 4550 \\
\hline I & SG20D03 & 1686.5 & 1689.8 & 15,815 & 15,867 & $13,390 \pm 170$ & 5649 \\
\hline I & SG20D04 & 1689.8 & 1693.0 & 15,867 & 15,926 & $13,020 \pm 80$ & 4551 \\
\hline $\mathrm{I}, \mathrm{II}$ & SG20D05 & 1693.0 & 1696.3 & 15,926 & 15,982 & $13,480 \pm 60$ & $5650 ; 8130$ \\
\hline I & SG20C03 & 1693.0 & 1696.3 & 15,926 & 15,982 & $13,630 \pm 50$ & $4552 ; 8128$ \\
\hline II & SG20C01 & 1702.8 & 1706.0 & 16,096 & 16,137 & $13,110 \pm 110$ & 5636 \\
\hline $\mathrm{I}, \mathrm{II}$ & SG20C02 & 1706.0 & 1709.3 & 16,137 & 16,183 & $13,610 \pm 70$ & 8134 \\
\hline I & SG20C05 & 1715.8 & 1719.0 & 16,298 & 16,351 & $13,890 \pm 80$ & 5651 \\
\hline I & SG20C06 & 1719.0 & 1722.3 & 16,351 & 16,408 & $13,860 \pm 130$ & 4553 \\
\hline $\mathrm{I}, \mathrm{II}$ & SG20B01 & 1726.0 & 1729.3 & 16,474 & 16,528 & $14,220 \pm 80$ & $6203 ; 8142$ \\
\hline I & SG20B02 & 1729.3 & 1732.5 & 16,528 & 16,577 & $13,820 \pm 70$ & 4554 \\
\hline
\end{tabular}


Table 1 Varve and ${ }^{14} \mathrm{C}$ chronologies of varved sediments from Lake Suigetsu. In the first column, I shows already reported data (Kitagawa and van der Plicht 1998a, 1998b); II and III show the new data measured in March, 1998 and August, 1998, respectively. Duplicate measurements are averaged. (Continued)

\begin{tabular}{|c|c|c|c|c|c|c|c|}
\hline \multirow[b]{2}{*}{ ID } & \multirow[b]{2}{*}{ Sample } & \multicolumn{2}{|c|}{ Depth $(\mathrm{cm})$} & \multicolumn{2}{|c|}{ Varve age (cal BP) } & \multirow{2}{*}{$\frac{{ }^{14} \mathrm{C} \text { age }}{(\mathrm{BP} \pm 1 \sigma)}$} & \multirow{2}{*}{$\begin{array}{l}\text { Lab code } \\
\text { GrA- }\end{array}$} \\
\hline & & Upper & Lower & Upper & Lower & & \\
\hline II & SG20B03 & 1732.5 & 1735.8 & 16,577 & 16,633 & $14,160 \pm 120$ & 8133 \\
\hline I & SG20B04 & 1735.8 & 1739.0 & 16,633 & 16,688 & $14,300 \pm 90$ & 5652 \\
\hline I & SG20A03 & 1755.3 & 1758.5 & 16,928 & 16,982 & $14,440 \pm 100$ & 4555 \\
\hline II & SG20A05 & 1761.8 & 1765.0 & 17,028 & 17,072 & $14,580 \pm 90$ & 8132 \\
\hline I & SG21D04 & 1779.8 & 1782.7 & 17,318 & 17,366 & $14,700 \pm 60$ & 4556 \\
\hline II & SG21D06 & 1785.7 & 1788.6 & 17,417 & 17,463 & $14,740 \pm 80$ & 8193 \\
\hline I & SG21D07 & 1788.6 & 1791.5 & 17,463 & 17,503 & $14,600 \pm 90$ & 4557 \\
\hline II & SG21D08 & 1791.5 & 1793.5 & 17,503 & 17,536 & $14,630 \pm 110$ & 8113 \\
\hline I & $\mathrm{SG} 21 \mathrm{C} 02$ & 1796.4 & 1799.3 & 17,582 & 17,631 & $14,630 \pm 60$ & 4558 \\
\hline I & $\mathrm{SG} 21 \mathrm{C} 03$ & 1799.3 & 1802.3 & 17,631 & 17,683 & $14,860 \pm 200$ & 4559 \\
\hline II & SG21C04 & 1802.3 & 1805.2 & 17,683 & 17,727 & $15,240 \pm 150$ & 8116 \\
\hline II & SG21C05 & 1805.2 & 1808.1 & 17,727 & 17,773 & $15,280 \pm 80$ & 8111 \\
\hline II & SG21C06 & 1808.1 & 1811.0 & 17,773 & 17,820 & $15,200 \pm 90$ & 8120 \\
\hline I & SG21C07 & 1811.0 & 1814.0 & 17,820 & 17,863 & $15,130 \pm 190$ & 4556 \\
\hline II & SG21B01 & 1814.0 & 1816.9 & 17,863 & 17,911 & $15,390 \pm 120$ & 8119 \\
\hline II & SG21B02 & 1816.9 & 1819.8 & 17,911 & 17,955 & $15,540 \pm 210$ & 8112 \\
\hline I & SG21B03 & 1819.8 & 1822.8 & 17,955 & 18,006 & $15,760 \pm 270$ & 4561 \\
\hline I & SG21B04 & 1822.8 & 1825.7 & 18,006 & 18,059 & $15,480 \pm 140$ & 5658 \\
\hline I & SG21B05 & 1825.7 & 1828.6 & 18,059 & 18,109 & $15,730 \pm 150$ & 5668 \\
\hline II & SG21B06 & 1828.6 & 1831.6 & 18,109 & 18,159 & $15,860 \pm 80$ & 8114 \\
\hline II & SG21A02 & 1837.4 & 1840.3 & 18,265 & 18,311 & $15,990 \pm 80$ & 8186 \\
\hline II & SG21A03 & 1840.3 & 1843.3 & 18,311 & 18,367 & $16,040 \pm 80$ & 8192 \\
\hline I & SG21A05 & 1846.2 & 1850.1 & 18,419 & 18,485 & $15,700 \pm 180$ & 4562 \\
\hline I & SG22D03 & 1862.1 & 1866.1 & 18,688 & 18,729 & $15,920 \pm 230$ & 4564 \\
\hline I & SG22D06 & 1872.7 & 1875.2 & 18,823 & 18,851 & $15,990 \pm 180$ & 4565 \\
\hline II & $\mathrm{SG} 22 \mathrm{C} 02$ & 1877.3 & 1880.3 & 18,880 & 18,929 & $16,350 \pm 90$ & 8124 \\
\hline II & $\mathrm{SG} 22 \mathrm{C} 03$ & 1880.3 & 1883.3 & 18,929 & 18,979 & $16,570 \pm 130$ & 8118 \\
\hline II & $\mathrm{SG} 22 \mathrm{C} 04$ & 1883.3 & 1886.4 & 18,979 & 19,036 & $16,700 \pm 130$ & 8123 \\
\hline I & SG22C06 & 1889.4 & 1894.5 & 19,083 & 19,179 & $16,280 \pm 200$ & 4566 \\
\hline II & $\mathrm{SG} 22 \mathrm{C} 07$ & 1894.5 & 1896.5 & 19,179 & 19,212 & $16,680 \pm 210$ & 8122 \\
\hline I & SG22B02 & 1899.5 & 1902.6 & 19,266 & 19,320 & $16,750 \pm 220$ & 5669 \\
\hline II & SG22B03 & 1902.6 & 1905.6 & 19,320 & 19,374 & $16,640 \pm 260$ & 8115 \\
\hline I & SG22B04 & 1905.6 & 1908.6 & 19,374 & 19,422 & $16,700 \pm 180$ & 5668 \\
\hline I & SG22B05 & 1908.6 & 1912.7 & 19,422 & 19,498 & $17,070 \pm 240$ & 4567 \\
\hline II & SG22B06 & 1912.7 & 1915.7 & 19,498 & 19,554 & $17,110 \pm 170$ & 8127 \\
\hline I & SG22A01 & 1917.7 & 1920.8 & 19,589 & 19,646 & $17,140 \pm 170$ & 4586 \\
\hline II & $\mathrm{SG} 22 \mathrm{~A} 03$ & 1924.8 & 1926.9 & 19,725 & 19,766 & $16,950 \pm 80$ & 8155 \\
\hline I & SG22A04 & 1926.9 & 1929.9 & 19,766 & 19,825 & $16,950 \pm 190$ & 4569 \\
\hline $\mathrm{I}, \mathrm{I}$ & SG22A05 & 1929.9 & 1932.9 & 19,825 & 19,883 & $17,140 \pm 90$ & $4570 ; 8187$ \\
\hline I & SG22A06 & 1932.9 & 1936.0 & 19,883 & 19,939 & $17,380 \pm 240$ & 5660 \\
\hline III & SG23D02 & 1943.2 & 1946.3 & 20,084 & 20,142 & $17,220 \pm 120$ & 10,245 \\
\hline I & SG23-4 & 1968.9 & 1969.8 & 20,588 & 20,606 & $17,750 \pm 140$ & 6193 \\
\hline III & SG23C04 & 1971.5 & 1974.6 & 20,636 & 20,684 & $17,200 \pm 180$ & 10,246 \\
\hline III & SG23C05 & 1974.6 & 1977.7 & 20,684 & 20,744 & $17,470 \pm 130$ & 10,247 \\
\hline III & $\mathrm{SG} 23 \mathrm{C} 07$ & 1980.9 & 1984.0 & 20,794 & 20,850 & $17,450 \pm 210$ & 10,269 \\
\hline
\end{tabular}


Table 1 Varve and ${ }^{14} \mathrm{C}$ chronologies of varved sediments from Lake Suigetsu. In the first column, I shows already reported data (Kitagawa and van der Plicht 1998a, 1998b); II and III show the new data measured in March, 1998 and August, 1998, respectively. Duplicate measurements are averaged. (Continued)

\begin{tabular}{|c|c|c|c|c|c|c|c|}
\hline \multirow[b]{2}{*}{ ID } & \multirow[b]{2}{*}{ Sample } & \multicolumn{2}{|c|}{ Depth $(\mathrm{cm})$} & \multicolumn{2}{|c|}{ Varve age (cal BP) } & \multirow{2}{*}{$\frac{{ }^{14} \mathrm{C} \text { age }}{(\mathrm{BP} \pm 1 \sigma)}$} & \multirow{2}{*}{$\begin{array}{l}\text { Lab code } \\
\text { GrA- }\end{array}$} \\
\hline & & Upper & Lower & Upper & Lower & & \\
\hline III & SG23B04 & 1993.4 & 1996.6 & 21,015 & 21,070 & $17,750 \pm 160$ & 10,249 \\
\hline III & SG23B06 & 1999.7 & 2002.9 & 21,123 & 21,173 & $17,430 \pm 200$ & 10,248 \\
\hline III & SG23A01 & 2006.0 & 2009.2 & 21,219 & 21,274 & $17,960 \pm 200$ & 10,270 \\
\hline III & SG23A03 & 2012.3 & 2015.4 & 21,332 & 21,379 & $18,240 \pm 230$ & 10,250 \\
\hline III & SG23A07 & 2024.9 & 2028.0 & 21,518 & 21,566 & $17,960 \pm 130$ & 10,252 \\
\hline III & SG24E01 & 2028.0 & 2031.0 & 21,566 & 21,622 & $17,970 \pm 130$ & 10,253 \\
\hline III & SG24E02 & 2031.0 & 2034.0 & 21,622 & 21,675 & $18,090 \pm 230$ & 10,254 \\
\hline I & SG24-5 & 2050.5 & 2051.6 & 21,961 & 21,979 & $18,810 \pm 110$ & 6192 \\
\hline III & SG24D03 & 2051.1 & 2054.1 & 21,972 & 22,027 & $18,770 \pm 130$ & 10,255 \\
\hline I & SG24-4 & 2053.8 & 2054.8 & 22,021 & 22,037 & $18,980 \pm 290$ & 6191 \\
\hline III & SG24D04 & 2054.1 & 2057.2 & 22,027 & 22,080 & $18,780 \pm 200$ & 10,383 \\
\hline III & SG24D05 & 2057.2 & 2060.2 & 22,080 & 22,136 & $18,830 \pm 150$ & 10,256 \\
\hline I & SG24-3 & 2064.9 & 2065.9 & 22,211 & 22,224 & $19,370 \pm 140$ & 6190 \\
\hline III & $\mathrm{SG} 24 \mathrm{C} 02$ & 2068.7 & 2071.7 & 22,273 & 22,325 & $18,930 \pm 450$ & 10,258 \\
\hline III & SG24B05 & 2094.9 & 2097.9 & 22,696 & 22,742 & $19,030 \pm 390$ & 10,262 \\
\hline III & SG24B08 & 2103.9 & 2105.9 & 22,840 & 22,877 & $19,190 \pm 130$ & 10,263 \\
\hline I & SG24-1 & 2106.4 & 2107.4 & 22,884 & 22,901 & $19,430 \pm 310$ & 6189 \\
\hline III & SG24A01 & 2105.9 & 2108.9 & 22,877 & 22,924 & $19,760 \pm 140$ & 10,261 \\
\hline III & SG25E05 & 2131.1 & 2134.1 & 23,280 & 23,331 & $19,810 \pm 200$ & 10,264 \\
\hline III & SG25E06 & 2134.1 & 2137.1 & 23,331 & 23,386 & $19,460 \pm 200$ & 10,265 \\
\hline III & SG25D01 & 2140.2 & 2143.2 & 23,441 & 23,494 & $20,040 \pm 210$ & 10,266 \\
\hline I & SG25-2 & 2149.0 & 2150.1 & 23,600 & 23,618 & $19,830 \pm 370$ & 6188 \\
\hline III & $\mathrm{SG} 25 \mathrm{C} 02$ & 2163.3 & 2166.3 & 23,833 & 23,885 & $20,110 \pm 200$ & 19,401 \\
\hline I & SG25-1 & 2175.0 & 2176.0 & 24,030 & 24,046 & $20,630 \pm 130$ & 6187 \\
\hline III & SG25C06 & 2175.3 & 2178.4 & 24,036 & 24,090 & $20,430 \pm 150$ & 10,361 \\
\hline III & $\mathrm{SG} 25 \mathrm{C} 08$ & 2181.4 & 2183.4 & 24,144 & 24,182 & $20,500 \pm 450$ & 10,362 \\
\hline III & SG25B03 & 2189.4 & 2192.4 & 24,301 & 24,347 & $20,540 \pm 560$ & 10,367 \\
\hline III & SG26D01 & 2210.0 & 2213.0 & 24,630 & 24,692 & $20,830 \pm 150$ & 10,360 \\
\hline III & SG26D03 & 2216.1 & 2219.1 & 24,750 & 24,813 & $21,060 \pm 150$ & 10,368 \\
\hline III & SG26C01 & 2234.3 & 2237.4 & 25,104 & 25,151 & $21,270 \pm 200$ & 10,404 \\
\hline III & SG26B03 & 2263.7 & 2266.7 & 25,581 & 25,627 & $22,060 \pm 260$ & 10,369 \\
\hline I & SG26-3 & 2264.8 & 2266.9 & 25,600 & 25,629 & $22,600 \pm 440$ & 6186 \\
\hline III & SG26B05 & 2269.8 & 2272.8 & 25,679 & 25,733 & $22,080 \pm 160$ & 10,370 \\
\hline I & SG26-2 & 2277.9 & 2278.9 & 25,803 & 25,818 & $22,630 \pm 220$ & 6185 \\
\hline III & SG26A01 & 2278.9 & 2281.9 & 25,819 & 25,859 & $22,280 \pm 160$ & 10,371 \\
\hline III & SG26A02 & 2281.9 & 2285.0 & 25,859 & 25,906 & $22,280 \pm 170$ & 10,372 \\
\hline I & SG26-1 & 2285.6 & 2286.6 & 25,915 & 25,932 & $23,170 \pm 150$ & 6184 \\
\hline III & SG26A03 & 2285.0 & 2288.0 & 25,906 & 25,954 & $22,230 \pm 390$ & 10,373 \\
\hline III & SG26A07 & 2297.2 & 2301.0 & 26,100 & 26,162 & $22,300 \pm 260$ & 10,375 \\
\hline I & SG27-7 & 2311.3 & 2312.3 & 26,336 & 26,351 & $23,400 \pm 500$ & 6183 \\
\hline I & SG27-5 & 2333.9 & 2334.9 & 26,696 & 26,714 & $24,500 \pm 270$ & 6182 \\
\hline I & SG27-4 & 2336.2 & 2337.3 & 26,742 & 26,757 & $23,890 \pm 210$ & 6181 \\
\hline I & SG27-3 & 2339.5 & 2340.6 & 26,790 & 26,819 & $23,970 \pm 170$ & 6180 \\
\hline I & SG27-2 & 2355.5 & 2356.5 & 27,047 & 27,061 & $24,600 \pm 270$ & 6179 \\
\hline I & SG28-4 & 2406.2 & 2407.2 & 27,803 & 27,821 & $24,700 \pm 270$ & 6178 \\
\hline I & SG28-3 & 2408.7 & 2409.7 & 27,847 & 27,862 & $25,130 \pm 190$ & 6177 \\
\hline
\end{tabular}


Table 1 Varve and ${ }^{14} \mathrm{C}$ chronologies of varved sediments from Lake Suigetsu. In the first column, I shows already reported data (Kitagawa and van der Plicht 1998a, 1998b); II and III show the new data measured in March, 1998 and August, 1998, respectively. Duplicate measurements are averaged. (Continued)

\begin{tabular}{|c|c|c|c|c|c|c|c|}
\hline \multirow[b]{2}{*}{ ID } & \multirow[b]{2}{*}{ Sample } & \multicolumn{2}{|c|}{ Depth $(\mathrm{cm})$} & \multicolumn{2}{|c|}{ Varve age (cal BP) } & \multirow{2}{*}{$\frac{{ }^{14} \mathrm{C} \text { age }}{(\mathrm{BP} \pm 1 \sigma)}$} & \multirow{2}{*}{$\begin{array}{l}\text { Lab code } \\
\text { GrA- }\end{array}$} \\
\hline & & Upper & Lower & Upper & Lower & & \\
\hline I & SG28-2 & 2433.0 & 2434.0 & 28,275 & 28,290 & $24,550 \pm 270$ & 6176 \\
\hline I & SG29-3 & 2508.6 & 2509.6 & 29,353 & 29,374 & $25,980 \pm 670$ & 6173 \\
\hline I & SG29-2 & 2537.7 & 2538.7 & 29,860 & 29,876 & $25,840 \pm 670$ & 6172 \\
\hline I & SG29-1 & 2560.5 & 2561.6 & 30,166 & 30,177 & $25,450 \pm 190$ & 6171 \\
\hline III & SG30D06 & 2600.9 & 2604.5 & 30,780 & 30,852 & $28,530 \pm 150$ & 10,381 \\
\hline III & SG30C01 & 2604.5 & 2607.6 & 30,852 & 30,905 & $28,140 \pm 290$ & 10,378 \\
\hline III & SG30C03 & 2610.7 & 2613.8 & 30,964 & 31,016 & $28,860 \pm 290$ & 10,380 \\
\hline III & SG30C04 & 2613.8 & 2616.8 & 31,016 & 31,081 & $28,770 \pm 230$ & 10,376 \\
\hline I & SG30-5 & 2622.9 & 2623.9 & 31,192 & 31,209 & $26,460 \pm 220$ & 6168 \\
\hline III & SG30C07 & 2623.0 & 2626.1 & 31,194 & 31,250 & $27,420 \pm 670$ & 10,395 \\
\hline III & SG30C10 & 2632.3 & 2635.3 & 31,355 & 31,411 & $27,520 \pm 720$ & 10,388 \\
\hline III & SG30B01 & 2635.3 & 2637.4 & 31,411 & 31,444 & $28,960 \pm 230$ & 10,389 \\
\hline I & SG30-4 & 2635.9 & 2636.9 & 31,421 & 31,438 & $27,880 \pm 240$ & 6169 \\
\hline III & SG30B05 & 2646.6 & 2649.2 & 31,578 & 31,617 & $29,450 \pm 190$ & 10,391 \\
\hline III & SG30A04 & 2658.5 & 2661.6 & 31,759 & 31,809 & $30,270 \pm 330$ & 10,390 \\
\hline I & SG30R-1 & 2661.0 & 2662.1 & 31,801 & 31,816 & $28,500 \pm 250$ & 6174 \\
\hline I & SG30-3 & 2662.9 & 2664.0 & 31,829 & 31,845 & $28,220 \pm 250$ & 6170 \\
\hline I & SG30-1 & 2671.2 & 2672.3 & 31,939 & 31,955 & $28,500 \pm 260$ & 6167 \\
\hline III & SG31-7 & 2697.8 & 2698.9 & 32,320 & 32,337 & $30,080 \pm 200$ & 5618 \\
\hline I & SG31D08 & 2698.8 & 2695.5 & 32,336 & 32,389 & $30,470 \pm 060$ & 10,415 \\
\hline III & SG31C02 & 2709.2 & 2712.5 & 32,505 & 32,559 & $31,310 \pm 770$ & 10,416 \\
\hline I & SG31-6 & 2716.0 & 2717.1 & 32,606 & 32,620 & $30,010 \pm 310$ & 5617 \\
\hline III & SG31C04 & 2715.8 & 2719.1 & 32,603 & 32,652 & $30,360 \pm 700$ & 10,417 \\
\hline I & SG31-5 & 2732.0 & 2733.1 & 32,834 & 32,850 & $31,550 \pm 340$ & 5616 \\
\hline I & SG31-4 & 2739.5 & 2740.6 & 32,969 & 32,989 & $31,550 \pm 340$ & 5615 \\
\hline III & SG31B05 & 2741.0 & 2744.3 & 32,995 & 33,047 & $31,750 \pm 810$ & 10,419 \\
\hline I & SG31-1 & 2751.3 & 2752.4 & 33,162 & 33,176 & $31,350 \pm 360$ & 5613 \\
\hline I & SG31-3 & 2760.9 & 2762.0 & 33,317 & 33,336 & $31,550 \pm 330$ & 5614 \\
\hline I & SG31-7 & 2764.1 & 2765.2 & 33,371 & 33,388 & $31,190 \pm 360$ & 5625 \\
\hline III & SG32F03 & 2770.0 & 2773.2 & 33,470 & 33,526 & $31,380 \pm 760$ & 10,422 \\
\hline I & SG32-6 & 2791.7 & 2792.7 & 33,853 & 33,871 & $32,140 \pm 260$ & 5624 \\
\hline III & SG32D02 & 2797.8 & 2801.0 & 33,943 & 33,996 & $33,980 \pm 740$ & 10,426 \\
\hline I & SG32-5 & 2800.1 & 2801.2 & 33,980 & 33,998 & $32,880 \pm 370$ & 5623 \\
\hline III & SG32C01 & 2804.2 & 2807.4 & 34,053 & 34,100 & $34,940 \pm 180$ & 10,429 \\
\hline I & SG32-4 & 2806.5 & 2807.5 & 34,086 & 34,101 & $32,830 \pm 380$ & 5622 \\
\hline III & SG32C02 & 2807.4 & 2810.7 & 34,100 & 34,144 & $34,500 \pm 470$ & 10,430 \\
\hline I & SG32-2 & 2812.8 & 2813.9 & 34,177 & 34,194 & $33,480 \pm 350$ & 5620 \\
\hline I & SG32-1 & 2855.1 & 2856.2 & 34,841 & 34,858 & $33,070 \pm 730$ & 5619 \\
\hline I & SG33-4 & 2913.3 & 2914.4 & 35,733 & 35,749 & $33,270 \pm 680$ & 5626 \\
\hline I & SG33-3 & 2920.1 & 2921.2 & 35,848 & 35,865 & $32,640 \pm 330$ & 5627 \\
\hline I & SG34-2 & 2976.0 & 2977.2 & 36,798 & 36,813 & $34,950 \pm 420$ & 5631 \\
\hline I & SG34-4 & 2993.7 & 2994.9 & 37,088 & 37,107 & $35,140 \pm 420$ & 5632 \\
\hline III & SG34B06 & 2994.3 & 2997.8 & 37,096 & 37,155 & $35,320 \pm 250$ & 10,434 \\
\hline I & SG34-3 & 3012.6 & 3013.7 & 37,392 & 37,417 & $35,070 \pm 460$ & 5633 \\
\hline
\end{tabular}

